First Peoples Child \& Family Review

An Interdisciplinary Journal Honouring the Voices, Perspectives, and Knowledges of First Peoples through Research, Critical Analyses, Stories, Standpoints and Media Reviews

\title{
L'intervention de groupe pour soutenir le passage à la vie adulte des jeunes autochtones issus des centres jeunesse et de deux communautés
}

\author{
Stéphane Grenier, Martin Goyette, Daniel Turcotte, Varda Mann-Feder and \\ Marie-Ève Turcotte
}

Volume 7, Number 2, 2013

URI: https://id.erudit.org/iderudit/1068847ar

DOI: https://doi.org/10.7202/1068847ar

See table of contents

Publisher(s)

First Nations Child and Family Caring Society of Canada

ISSN

1708-489X (print)

2293-6610 (digital)

Explore this journal

Cite this article

Grenier, S., Goyette, M., Turcotte, D., Mann-Feder, V. \& Turcotte, M.-̇̀. (2013).

L'intervention de groupe pour soutenir le passage à la vie adulte des jeunes

autochtones issus des centres jeunesse et de deux communautés. First Peoples

Child \& Family Review, 7(2), 148-159. https://doi.org/10.7202/1068847ar

Copyright (c) Stéphane Grenier, Martin Goyette, Daniel Turcotte, Varda Mann-Feder, Marie-Ève Turcotte, 2013
This document is protected by copyright law. Use of the services of Érudit (including reproduction) is subject to its terms and conditions, which can be viewed online.

https://apropos.erudit.org/en/users/policy-on-use/ 


\title{
First Peoples Child \& Family Review
}

An Interdisciplinary Journal Honoring the Voices, Perspectives and Knowledges of First Peoples through Research, Critical Analyses, Stories, Standpoints and Media Reviews

Volume 7, Number 2, 2013, pp. 148-159

\section{L'intervention de groupe pour soutenir le passage à la vie adulte des jeunes autochtones issus des centres jeunesse et de deux communautés}

\author{
Varda Mann-Feder ${ }^{d}$ and Marie-Ëve Turcotte ${ }^{b}$ \\ a Université du Québec en Abitibi-Témiscamingue \\ b École nationale d'administration publique \\ c Université Laval \\ d Université Concordia
}

Stéphane Greniera, Martin Goyette ${ }^{\mathrm{b}}$, Daniel Turcottec,

Le passage à la vie adulte des jeunes perçus comme "vulnérables » ou " en difficulté » est une préoccupation grandissante à la fois pour les praticiens et les décideurs publics (Goyette \& Turcotte, 2004). On cherche par tous les moyens de les soutenir. Ces jeunes ont en effet tendance à être surreprésentés dans les populations marginales (ex. itinérants, toxicomanes, contrevenants, etc...) (La Prairie \& Stenning, 2003; Tweddle, 2007; Guay \& Grammond, 2010). Malgré de nombreuses études qui vantent les mérites de programmes ou d'interventions fondés sur la modification des attitudes et des comportements par l'apprentissage social et la transmission de connaissances (Coren et al., 2003; Harris \& Franklin, 2003; Kissman, 1990; McDonell, Limber \& Connor-Godbey, 2007), bon nombre de jeunes " en difficulté " rencontrent des difficultés d'insertion sociale et professionnelle au début de lâge adulte (Goyette et Turcotte, 2004).

La littérature scientifique portant plus spécifiquement sur les conditions d'insertion sociale des jeunes autochtones révèle qu'ils doivent composer avec des obstacles qui leur sont particuliers tels que leur appartenance culturelle (Bousquet, 2005) et l'héritage des pensionnat (Graham, 2001). Au-delà d'une fierté d'appartenance à leur culture, les jeunes autochtones ont de la difficulté à arrimer leur appartenance culturelle à leur mode de vie contemporain, à se définir simultanément comme citoyen et autochtone (Bergeron, 2006). Outre le fait de se sentir tiraillés entre deux systèmes de valeurs, le manque de connaissance de soi et le peu de modèles d'identification qui s'offrent aux jeunes autochtones sont des facteurs qui inhibent leur mise en projet ${ }^{1}$ à lécole et dans le monde du travail (Bergeron, 2006). Le modèle de la vie autonome véhiculé dans la littérature nord-américaine s'inscrit en décalage avec la perspective autochtone sur la transition à la vie adulte (Long et al., 2006) et laisse les intervenants

Corresponding author:

Stéphane Grenier en service social avec peu de moyens pour aider stephane.grenier@uqat.ca

1 Leur intégration, leur réussite ou plus simplement leur cheminement. 
First Peoples Child \& Family Review, Volume 7, Number 2, 2013

Il s'agit là de constats préoccupants pour les organismes prestataires de services à la jeunesse, qui narrivent pas à soutenir ces jeunes de façon optimale dans leur processus d'insertion sociale. Il est donc impératif de tout mettre en œuvre pour aider les jeunes vulnérables et particulièrement les jeunes autochtones à franchir ce passage, particulièrement dans le cas des jeunes qui sont moins bien outillés, comme c'est le cas des jeunes issus des centres jeunesse ${ }^{2}$ qui voient les services se terminer abruptement à l'âge de 18 ans après une mesure de placement.

Ce texte porte sur l'expérimentation d'un programme d'intervention, D'hier à demain, axé sur la préparation au passage à la vie adulte de jeunes autochtones ayant reçu des services des centres jeunesse (Grenier et al., 2010). Il relate les étapes de conception, d'implantation et d'évaluation de ce programme basé sur l'utilisation de la méthode de groupe. Le texte se divise en trois parties. La première fait état des défis que pose le passage à la vie adulte pour les jeunes autochtones vulnérables. La seconde partie présente le processus et les éléments qui ont servi au développement du programme D'hier à demain. La troisième partie relate le processus d'expérimentation du programme auprès des jeunes autochtones qui ont connu une trajectoire de services en centre jeunesse.

\section{LES DÉFIS DU PASSAGE À LA VIE ADULTE}

Comme le souligne Bidart (2006), la modification des conditions d'entrée dans la vie adulte observée depuis quelques décennies est associée à l'allongement de la jeunesse et à une désynchronisation des moments où se déroulent les transitions à la vie adulte. Le passage à la vie adulte prend maintenant la forme d'un processus qui sétire dans le temps et qui, pour plusieurs jeunes, nécessite un soutien. Par ailleurs, les parcours d'entrée dans la vie adulte se sont diversifiés et ils sont maintenant marqués par des processus d'allers-retours et d'essais-erreurs (Charbonneau, 2004; Gauthier, 2000; Goyette et al., 2006; Goyette \& als., 2010). Également, le passage à la vie adulte ne se réalise plus selon un modèle-type (occupation d'un emploi mariage - achat d'une maison - enfant). Devenir adulte aujourd'hui veut non seulement dire franchir des étapes d'adaptation concrète, mais aussi effectuer sur le plan symbolique un travail de construction identitaire.

Dans le cas particulier des jeunes autochtones, selon Bousquet (2005), le défi particulier auquel ils font face aujourd'hui est de redéfinir une nouvelle façon dêtre autochtone en contexte de réserve et hors réserve. Elle dénote d'ailleurs une intention des jeunes algonquins ${ }^{3}$ de concilier les cultures autochtone et allochtone, en prenant garde, toutefois, de ne pas "virer blancs ». Ces jeunes ne s'inscrivent pas dans un processus de définition d'une double-identité, mais tentent au contraire de se construire une nouvelle identité autochtone, en empruntant à la fois à la tradition et à la modernité (Girard \& Lutumba, 2004).

En opposition avec l'objectif d'autonomisation des jeunes en général, la culture autochtone traditionnelle se fonde sur une vision du monde qui valorise l'interdépendance du jeune et de la communauté (Long et al., 2006; Goyette et al., 2008). En ce sens, la capacité de maintenir un lien et dêtre un soutien pour sa famille et sa communauté tout au long de sa vie est centrale pour les jeunes autochtones (Graham, 2001 ; Long et al., 2006; Munsell, 2004; Goyette et als. 2010). La transition à la vie adulte de ces jeunes est profondément marquée par le contraste des cultures autochtone et allochtone qui définissent différemment les critères d' " accomplissement adulte »

2 Les centres jeunesse sont les organismes chargés de l'application québécois de la loi sur la protection de la jeunesse.

3 Les algonquins sont une des dix nations autochtones du Québec.

(c) Stéphane Grenier, Martin Goyette, Daniel Turcotte, Varda Mann-Feder and Marie-Ève Turcotte 


\section{L'intervention de groupe pour soutenir le passage à la vie adulte des jeunes autochtone}

et les conditions pour y parvenir, notamment en regard de la poursuite des études et de la réussite scolaire (Bousquet, 2005; Fox et al., 2005).

La prise en compte du conflit identitaire est donc un incontournable dans analyse des enjeux du passage à la vie adulte des jeunes autochtones et dans lélaboration de modalités d'intervention des travailleurs sociaux pour soutenir ce passage.

\section{LA CONCEPTION DU PROGRAMME}

Pour accompagner des jeunes autochtones dans leur passage à la vie adulte, tout en reconnaissant cet écart entre les deux cultures, un modèle d'intervention de groupe auprès de la culture amérindienne, où la réalité est généralement prise en compte selon une perspective holistique qui entrevoit le monde à partir des connexions entre les personnes et les communautés (Stewart, 2009), a été développé. Le programme d'intervention de groupe construit dans le cadre de ce projet s'inspire de trois programmes visant la préparation au passage à la vie autonome, soit le programme Qualification des jeunes, Droit devant et Moi et Cie (Grenier et al, 2010). Des éléments tirés de ces programmes ont été adaptés pour tenir compte du concept de la roue médicinale (Loiselle, 2009), concept ancestral des populations amérindiennes et de l'entraide mutuelle en travail social.

Le programme Qualification des jeunes (PQJ) contribue à outiller les jeunes afin de développer des habiletés fonctionnelles, les préparer et les encadrer de manière à ce qu'ils puissent acquérir des compétences de la vie quotidienne, en plus de permettre de développer un réseau de soutien par l'entremise de diverses ressources (Association des centres jeunesses du Québec, 2011). Cependant, les habiletés fonctionnelles visées par le programme D’hier à demain sont davantage reliées à la culture et au mode de vie des jeunes autochtones.

La particularité du programme D’hier à demain (Grenier et al., 2010) réside avant tout dans la volonté d'offrir un contenu adapté sur le plan culturel, soit un contenu qui tient compte de la réalité de vie quotidienne des jeunes amérindiens et des questions qui les touchent plus particulièrement au moment de leur passage à la vie adulte. Ce programme se fonde sur l'aide mutuelle (Grenier et al., 2010). En travail social de groupe, ce modèle renvoie à une « utilisation holistique du groupe et reconnaît la valeur des forces des membres pour leur venir en aide » (Moyse Steinberg, 2008, p.34).

En proposant une intervention de groupe adapté à la réalité autochtone et plus près de leur conception du devenir adulte, le programme D'hier à demain offre une formule innovatrice aux intervenants qui désirent accompagner les jeunes autochtones dans leur passage à la vie adulte, cette période caractérisée par de multiples transitions. Ce programme a pour buts de permettre à des jeunes autochtones d'explorer différentes facettes du passage à la vie adulte et les amener à exprimer leurs désirs, leurs besoins et leurs craintes face à cette transition. Le programme est élaboré de façon à aider les jeunes autochtones à faire des prises de conscience personnelles et collectives par l'entremise de diverses activités jumelant la culture autochtone et occidentale. Les thèmes abordés dans le cadre du programme sont le rapport à soi et à ses origines, les projets résidentiels et professionnels, l'affirmation de soi, le réseau social et le soutien, les ressources environnantes, la contribution à sa communauté et l'accomplissement adulte. Pour ce faire, les activités proposées en lien avec ces thèmes tentent de s'insérer à l'intérieur de chacune des quatre dimensions de la roue médicinale, soit l'aspect spirituel, physique, émotif-relationnel et mental. 


\section{L'IMPLANTATION DU PROGRAMME}

Pour documenter la façon dont le programme D’hier à demain a été implanté, deux sources d'information ont été utilisées : les jeunes qui ont participé et les intervenants. Les participants ont été rencontrés en entrevue individuelle à deux moments, soit avant le début des interventions et après la dernière rencontre. Lors de ces entrevues, ils ont été interrogés sur la façon dont ils avaient été recrutés, sur leur motivation à participer au programme, sur leurs attentes par rapport au groupe et sur leur appréciation du déroulement des activités.

Les intervenants ont été rencontrés au terme du programme. Ils ont été invités à faire part de leur vision de la mise en œuvre des activités et de leur appréciation des effets produits par l'intervention. Ces entretiens réunissaient les deux intervenants chargés du groupe.

Les commentaires des intervenants et des jeunes ont été regroupés en fonction de trois aspects liés à son implantation: (1) l'engagement des jeunes dans les groupes, (2) le respect de l'approche par les intervenants (aide mutuelle), et (3) la place du programme D'hier à demain dans les pratiques des centres jeunesse.

\subsection{La formation des intervenants}

Afin de préparer l'implantation du programme dans deux communautés autochtones, deux journées de formation ont été offertes en octobre 2010. Ces formations ont porté sur les particularités de l'intervention en contexte autochtone, sur les principes de l'animation de groupe et sur l'utilisation de l'aide mutuelle. Onze intervenants provenant d'une diversité de milieux d'intervention y ont été conviés par une personne-ressource du Centre jeunesse de l'AbitibiTémiscamingue. Les participants ont reçu un manuel de formation et d'intervention (Grenier et al., 2010) résumant les concepts-clés relatifs à l'intervention de groupe dans une perspective d'aide mutuelle. À la suite de cette formation, quatre intervenants ont été identifiés pour prendre en charge les groupes d'intervention, ils ont été pairés dans des équipes mixtes composées d'un intervenant du centre jeunesse et d'un intervenant rattaché à un organisme communautaire basé sur chacune des communautés. Une rencontre de mi-parcours a eu lieu avec les quatre intervenants en charge des groupes, ainsi qu'avec quelques partenaires qui désiraient suivre le processus d'implantation de près. Le but de cette rencontre était de permettre aux intervenants d'échanger entre eux à propos du déroulement des groupes, des obstacles rencontrés et des éléments qui constituaient pour eux des succès. La rencontre était aussi l'occasion de leur offrir de la supervision clinique. En tout temps durant la mise en place des groupes, le formateur est demeuré disponible pour offrir de la supervision. Cependant, les intervenants n'ont pas eu à faire appel à ses services.

\subsection{Le recrutement}

Le recrutement des jeunes pour la participation aux groupes a été assuré par les intervenants responsables de leur mise en œuvre. Conformément aux critères de sélection déterminés par l'équipe de recherche en collaboration avec les comités de soutien à l'implantation pour faire partie du programme, les jeunes devaient être âgés entre 16 et 21 ans, avoir reçu des services des Centres jeunesse et être autochtone. Ils devaient être volontaire et pouvoir se rendre disponible pour les groupes. Il est à noter que les jeunes sollicités étaient pour la plupart déjà connus des intervenants en charge du recrutement. 


\section{L'intervention de groupe pour soutenir le passage à la vie adulte des jeunes autochtone}

\subsection{Les groupes}

Le programme D'hier à demain s'est déroulé à l'hiver 2011 auprès de deux groupes mixtes de jeunes adultes (garçons et filles confondus) provenant de deux communautés autochtones. Dans le premier groupe, sept jeunes ont participé au programme, dont six filles et un garçon. Lâge moyen des participants était de 19,1 ans. La plupart des participants vivent toujours chez leurs parents. Environ la moitié de ces jeunes avaient un emploi et la plupart était célibataires. Le niveau de scolarité est faible, la plupart des jeunes n'ayant pas complété la deuxième année du secondaire. Toutefois, la majorité des jeunes fréquente toujours l'école aux adultes dans des cheminements particuliers. Il faut noter que plusieurs participants, soit environ la moitié, sont parents d'un enfant. En raison de la distance géographique entre Montréal, où est basée l’équipe de recherche, et l'Abitibi, nous n'avons pas eu d’accès prolongé au terrain, ce qui nous a occasionné des contraintes importantes lorsqu'est venu le temps de rencontrer les jeunes pour les entretiens post-tests (après l'expérimentation du groupe). Nous n'avons donc pu rejoindre que deux jeunes de ce groupe pour compléter un second entretien de recherche dans les délais prescrits. ${ }^{4}$

Dans le deuxième groupe, cinq jeunes avaient été sélectionnés comme participants, dont deux filles et trois garçons. Lâge moyen des jeunes de ce groupe est de 18,6 ans. Tous les participants demeurent chez leurs parents et sont sans emploi. Seulement une participante a décroché de l'école et les quatre autres fréquentent l'école aux adultes. Trois participants sont célibataires, tandis que deux sont en couple. Le niveau de scolarité des jeunes de ce groupe est similaire au groupe précédent. Ce groupe compte moins de parents comparativement au premier groupe. Des cinq jeunes de ce groupe, trois ont participé aux entretiens post-tests.

Sans être en mesure d'affirmer que les échantillons des deux groupes (12 jeunes adultes) de jeunes sont représentatifs de chaque communauté (seulement cinq jeunes pour le premier groupe et quatre jeunes pour le deuxième groupe ont complété l'outil au premier temps de mesure), les résultats apparaissent cohérents en regard de certains éléments du contexte propre à chaque communauté et observé par les chercheurs et les intervenants en charge des groupes (Goyette et al, 2012). Dans le souci de préserver l'identité de chaque communauté, nous nous contenterons simplement de mentionner que le premier groupe a été réalisé dans une communauté qui se situe plus loin de la ville, qui comporte beaucoup moins d'habitants et où les conditions de vie sont plus rudimentaires. ${ }^{5}$ Quoique cette hypothèse demande à être confirmée par des recherches ultérieures, il est fort probable que l'ensemble de ces conditions favorise, pour le premier groupe, la consolidation d'une identité culturelle qui se distingue davantage de la culture dominante.

\section{L'ÉVALUATION DES RÉSULTATS}

\subsection{Une approche culturellement adaptée}

Le programme D'hier à demain (Grenier et al, 2010) se voulait avant tout une initiative culturellement adaptée. ${ }^{6}$ Lapproche qui sous-tend le programme ainsi que sa banque d'activités ont été pensées en fonction de cette préoccupation. Du point de vue de plusieurs intervenants, lapproche ouverte et participative qui sous-tend le groupe D'hier à demain a constitué une

4 Ceci limite notre possibilité de voir les changements sur les jeunes à long termes.

5 Sans électricité et eau courante.

6 Par culturellement adaptée, nous voulons dire qui tenait compte des différences culturelles. 
First Peoples Child \& Family Review, Volume 7, Number 2, 2013

formule gagnante en contexte autochtone. Les intervenants soulignent à cet effet que les jeunes ont particulièrement bien réagi aux moments de "déstructuration relative» ${ }^{7}$ que permettait l'approche, et même qu'ils les ont appréciés.

Du point de vue de certains intervenants, les jeunes autochtones sont aussi plus à même de réaliser des apprentissages à partir d'activités qui leur permettent de cheminer à leur rythme. Lidée du " groupe par le groupe ", à la base des approches mises de l'avant par le programme expérimenté, permet justement cette ouverture.

" Je compare ça un petit peu à combien de jeunes autochtones sont aux études aux adultes mais qu'ils peuvent aller à leur rythme, en écoutant de la musique, plus sweet justement, tranquilles. Je vais à mon rythme puis je finirai quand je finirai. Je trouve que le groupe par le groupe c'est un petit peu ça aussi, en disant on commence, on ne sait pas comment ça va virer, on ne sait pas où on va aller exactement, mais on va les suivre puis on va s'adapter. C'est similaire un petit peu. » (Intervenant)

"C’est qu’on faisait genre qu'est-ce qu’on voulait. On nétait pas à l'école.» (Jeune participant)

Il appert donc que le cheminement personnel des jeunes autochtones a été favorisé par la dynamique de partage authentique avec les autres membres du groupe, incluant les intervenants. Ce principe est fondamental dans le cadre d'un groupe mené dans l'optique de l'aide mutuelle, l'actualisation de dynamiques d'entraide témoignant d'une implantation réussie de l'approche.

Lapproche de l'aide mutuelle s'est avérée particulièrement gagnante en contexte autochtone, parce qu'elle s'inscrit en cohérence avec les normes culturelles de non-interférence. Elle ouvre également vers la possibilité de créer un lien entre jeunes et intervenants sur la base d'un rapport égalitaire et significatif, en conformité avec les normes culturelles. Elle présente toutefois un défi supplémentaire lorsqu’elle est implantée dans les communautés caractérisées par une grande proximité physique et relationnelle de ses membres. ${ }^{8}$

\subsection{Des activités non culturellement adaptées?}

Les activités qui évoquaient des éléments de culture traditionnelle (ex. cérémonie de la sauge, discuter autour d'une légende, découvrir son animal totem) n’ont pas été particulièrement bien reçues par les jeunes. Plusieurs jeunes ont confié que ces activités ne les intéressaient pas et qu'ils n’en comprenaient pas le but. ${ }^{9}$

« R- La première rencontre c’était genre une histoire. I- Cétait une légende, c’est ça. R- Oui cétait une légende puis... I- C’était plus genre sur la culture autochtone ou quelque chose de même? R- Oui. Ça... I- Moins. R- Je nétais pas très attentive à ça. I- C'est quoi qui ténervait dans ça? R- Ben je ne comprenais pas l'histoire. » (Jeune participant)

Un jeune mentionne quant à lui que ce n'est pas le rôle des intervenants allochtones de transmettre la culture traditionnelle aux jeunes autochtones. On comprend facilement, à la lecture de son témoignage, son désir que ce soit les autochtones eux-mêmes qui se réapproprient leur culture.

\footnotetext{
7 Ce concept renvoie au moment où l'intervention n'est pas planifiée à l'avance, mais où elle se construit dans l'instant présent des interactions.

8 Ce qui se passe dans le groupe a nécessairement des impacts rapide à l'extérieur du groupe dans ce genre de communauté.

9 Cela nous renvoie aux travaux de Bousquet (2005) sur l'identité culturelle des jeunes autochtones. Pris entre deux cultures, ils développent leur propre culture qui n'est pas nécessairement celle que leur ainés souhaiteraient.
}

(c) Stéphane Grenier, Martin Goyette, Daniel Turcotte, Varda Mann-Feder and Marie-Ève Turcotte 


\section{L'intervention de groupe pour soutenir le passage à la vie adulte des jeunes autochtone}

« Mettons la culture autochtone c'est mes parents, ma mère ou ben mes grands-parents. C'est eux autres peut-être qui mapprendraient plus tout qu'est-ce qui est histoire ou des affaires de même, l'histoire autochtone ou ben la trappe, des affaires de même.(...) Cétait important mais je veux dire ce qu'ils nous apprennent... en tout cas je ne sais pas comment je pourrais dire ça... Eux autres c'est des Blancs, ça fait que je trouve que des Blancs pourquoi qu'ils viendraient nous apprendre la culture. » (Jeune participant)

Par ailleurs, plusieurs jeunes ont témoigné de leur malaise à porter la culture traditionnelle; ils ont exprimé leur désir de dépasser les clichés et de redéfinir et d'actualiser l'identité de jeune autochtone. Ainsi, la composante identitaire du programme est-t-elle des plus pertinentes, mais nous constatons qu’elle a été plutôt mal abordée dans le cadre du programme.

\subsection{Vivre des expériences et bouger}

les deux équipes d'intervenants ont relevé que les activités prévues au programme d'intervention de groupe devaient permettre aux jeunes autochtones de bouger et de vivre des expériences pour susciter leur intérêt. Ils ont donc adapté plusieurs activités pour les rendre plus stimulantes pour les jeunes sur le plan de la mise en action. Il s'agit de faire bouger les jeunes et non pas seulement de discuter ou de faire des activités plus passives, comme réaliser un bricolage ou regarder un film.

« On le sait qu’avec les jeunes autochtones ici ce qui marche c'est tout ce qui est tactile, visuel, c'est de les mettre beaucoup plus en action. (...) On s'est vraiment adaptés pour les faire participer au maximum. Là cétait les mises en situation. » (Intervenant)

À ce sujet, il est intéressant de constater que le groupe a été perçu par plusieurs jeunes autochtones comme une occasion de contrer l'inactivité. Les communautés sont décrites par les jeunes comme des lieux où il n'y a pas grand chose de constructif à faire. Ils ont donc apprécié l'opportunité que représente le groupe au niveau de la mise en action.

\section{Impacts du groupe sur les jeunes}

\subsection{Espace de prises de conscience}

Selon le discours des jeunes autochtones, l'effet principal que le groupe a eu sur eux consiste en la réalisation de prises de conscience. Ainsi, le groupe est avant tout perçu comme une opportunité d' " élargir ses horizons » et " d'ouvrir des voies ».

« Disons que ça me servait à m’amener d’autres expériences, de voir d’autres frontières que celles que jai aujourd'hui. Ça élargit mes horizons. » (Jeune participant)

Les jeunes autochtones voient le groupe comme une occasion privilégiée d'être confrontés à différentes opinions et de prendre le temps de les considérer. Les jeunes autochtones ont démontré une ouverture plus grande devant les opportunités qu'offrait le groupe de se questionner sur leurs croyances, leurs comportements et leurs valeurs.

«I- Tu pourrais-tu me donner un exemple de qu'est-ce que ça pourrait être les changements? R- Ça pourrait être que je pourrais être un peu plus ouvert d'esprit sur les opinions des autres. I- Oui? R- Oui avant je ne faisais pas ça. Avant cétait juste mon opinion puis that's it, that's all. (...) C’était vraiment javais une idée... I- Chacun son idée. R- Chacun son idée puis... 


\section{First Peoples Child \& Family Review, Volume 7, Number 2, 2013}

I- Personne ne vous avait jamais fait partager dans le fond vos idées? R- Non, cétait vraiment chacun notre idée puis si tu es pas d’accord avec mon idée ben tu vas jouer là-bas. » (Jeune participant)

\subsection{Créer des liens}

Le groupe se présente comme un espace de création de liens, à la fois du point de vue des jeunes et des intervenants. C'est d'abord une occasion pour les jeunes de créer des liens avec d’autres jeunes, ou encore, de renforcer les relations qui existent déjà entre eux. À cet effet, certains jeunes soulignent à quel point ils ont apprécié l'opportunité de se rencontrer pour parler de sujets qu'ils n’avaient jamais encore évoqués ensemble.

«R- Mettons comme dans les rencontres, il y avait des trucs plus personnels. Je ne sais pas comment dire ça. Ils n’en parlent pas mais... on n’en parle pas... I- Vous n’aviez jamais parlé de ça? R- Non. I- Ça cétait tu quelque chose de positif mettons d’amener des sujets que... R- Oui. Mon ami il venait, on dirait que je le connais plus. » (Jeune participant)

Le groupe est aussi une occasion pour les jeunes et les intervenants de réparer un lien fragilisé et souvent caractérisé à la base par un manque de confiance mutuel. Les différentes activités ont permis aux jeunes autochtones et aux intervenants d'apprendre à se faire confiance et à s'entraider. Ainsi, le groupe a représenté un espace d’entraide pour bon nombre de jeunes. Ces derniers ont notamment pu se confier entre eux à propos de leurs difficultés, et ils disent sêtre sentis compris et acceptés dans ce partage.

« J'ai trouvé ça le fun, on s'entendait pis il y a des affaires que je vivais que les autres vivaient, ça fait qu'on a pu s'en parler. (...) J'aime pas ça parler de moi avec des gens plus vieux ou des gens comme mes parents. Mais ici il y a des gens qui me comprennent pis ils sont comme « Oui moi aussi jai vécu ça. » (Jeune participant)

Le groupe lie les participants entre eux. Cette cohésion fonctionne comme le moteur du groupe, à tel point que le départ inattendu d'un participant peut être vécu comme une expérience de rejet ou d'abandon par d'autres membres du groupe.

«Un des participants a lâché prise comme à la troisième rencontre. Ça fait que ça nả pas aidé. (...) Ben on était un groupe pis on se tenait, pis lui il a décidé de sacrer son camp. » (Jeune participant)

\subsection{Espace d'appropriation}

De façon plus évidente pour les jeunes ayant vécu un placement en centre jeunesse, le groupe se présente comme un espace d'appropriation. Ces derniers mentionnent que dans le contexte du placement, ils ont généralement peu de latitude pour s’exprimer librement. On fait peu de place à leur participation dans les décisions qui les concernent.

"C’était différent parce que là cétait notre place pour parler dans le fond. Les autres groupes mettons que cétait juste que tu avais des choses à suivre, mais là c’était notre place pour parler. (...) Cétait comme un collectif. (...) On se partageait des affaires pis ça restait confidentiel. » (Jeune participant)

Si le groupe est un espace d'appropriation au sens où il donne la parole aux jeunes, il peut aussi lêtre en permettant aux jeunes de se construire en se positionnant personnellement par 


\section{L'intervention de groupe pour soutenir le passage à la vie adulte des jeunes autochtone}

rapport aux enjeux qui les concernent. Or, cet effet est surtout observé dans les groupes où les intervenants se sont dits plus à l'aise à mettre en œuvre les approches de la facilitation (Schwarz et al., 2005) et de l'aide mutuelle (Moyse Steinberg, 2008).

En effet, la facilitation est un processus à travers lequel une personne qui est acceptée par tous les participants, qui est neutre et qui n'a pas de pouvoir particulier sur le groupe, intervient pour les aider à améliorer la façon selon laquelle ils identifient les problèmes, les résolvent et prennent des décisions (Schwarz et al., 2005). L'aide mutuelle quant à elle apparait spontanément dans les groupes s'il y a assez d'espace de disponible pour faciliter les échanges constructif (Moyse Steinberg, 2008).

\subsection{Espace de valorisation}

Le groupe apparaît par ailleurs comme un espace de valorisation pour les jeunes. À travers l'ensemble des activités réalisées, les jeunes confient avoir vécu des réussites, ce qui a contribué à favoriser leur estime de soi.

Le parcours dans le groupe a aussi permis à certains jeunes de se sentir davantage en mesure de faire face aux défis qui les attendent et plus confiants par rapport à leurs perspectives d'avenir. Le groupe a conduit ces jeunes à envisager l'avenir avec davantage d'espoir et de confiance, en les amenant à considérer les possibilités plutôt que les obstacles.

« Je me sens plus confiant envers moi-même aussi pour le futur, même si jai encore des zones ombragées, des zones nébuleuses. Mais je me sens plus confiant pour aller droit devant. » (Jeune participant)

Le groupe a aussi été l'occasion pour certains jeunes non seulement de se prouver à eux-mêmes qu'ils étaient capables d'affronter les défis qui les attendent, mais aussi d'en faire la démonstration aux intervenants présents dans leur vie et aux personnes de leur entourage.

\section{CONCLUSION}

En terminant, à la lumière de cette étude, les programmes d'intervention de groupe fondés sur l'approche d'aide mutuelle, comme le programme D'hier à demain, devraient être intégrés au curriculum régulier d'interventions destinées aux jeunes en transition à la vie adulte. Ce type de programme permet aux jeunes de s'autonomiser par le vécu d'expériences concrètes, de soutenir leur construction identitaire et de réparer le lien parfois précaire entre les jeunes et les organismes et établissements prestataires de services sociaux. Lanalyse thématique du discours des jeunes participants et des intervenants met en valeur que le programme d'Hier à Demain a permis l'émergence de dynamiques de groupe constructives pour les jeunes autochtones, dans le sens où elles soutiennent leur cheminement vers l'autonomie. Le groupe est un espace d'apprentissage et de conscientisation, d'appropriation, de création de liens, de valorisation et d'appartenance.

Il est impératif de considérer à la fois les jeunes et les intervenants comme des acteurs de premier plan dans le processus de transformation des pratiques. C'est à partir des jeunes que les interventions doivent être pensées, dans le respect de leur volonté et de leur autonomie. Cette façon d'envisager la pratique, telle que soutenue par cette recherche, répond à une conception de l'efficacité, qui renvoie davantage à l'idée d'une adéquation entre les interventions mises en œuvre et les besoins réels des communautés cibles. 
First Peoples Child \& Family Review, Volume 7, Number 2, 2013

Les résultats de cette expérience indiquent qu'une approche d'intervention de groupe axée sur l'aide mutuelle est susceptible de rejoindre plus facilement les jeunes qui se montrent plutôt réfractaires aux approches plus directives. En effet, l'expérience de groupe a été perçue par plusieurs jeunes comme différente des interventions habituelles qui sont souvent critiquées par les jeunes. De par son contraste avec le caractère normatif, directif et parfois " ennuyeux » des interventions menées en contexte d’autorité qui sollicitent peu les jeunes dans la définition des orientations à prendre et des activités à réaliser, l'intervention de groupe qui mise sur une participation accrue des jeunes permet manifestement, de les placer dans une position plus intéressante en leur redonnant du pouvoir dans tout le processus.

En plus de contribuer à développer des liens particulièrement étroits entre eux et de les amener à effectuer un travail en profondeur sur eux-mêmes, le groupe axé sur l'aide mutuelle s'est révélé une excellente tribune où les jeunes autochtones ont pu mettre à profit leur autodétermination et briser leur oisiveté.

Dans la perspective de toujours travailler à une meilleure adaptation du programme sur le plan culturel, les activités du programme d'Hier à demain devraient être conçues pour faire bouger les jeunes autochtones encore davantage, de façon à susciter leur intérêt, et pour favoriser les prises de conscience et les apprentissages par le groupe.

\section{RÉFÉRENCES}

Association des centres jeunesse du Québec (ACJQ). (2011). Le programme PQJ. Consulté le 20 avril 2011, from http:// www.acjq.qc.ca/?3EC45ADF-FDAC-4091-B1E8-668194B820FA

Bergeron, S. (2006). Le conflit identitaire et l'insertion socioprofessionnelle des jeunes autochtones. Université Laval, Québec.

Bidart, C. (2006). Devenir adulte aujourd'hui : perspectives internationales: INJEP, Collection débats-Jeunesse, L'Harmattan.

Bousquet, M.-P. (2005). Les jeunes Algonquins sont-ils biculturels? Modèles de transmission et innovations dans quelques réserves. Recherches amérindiennes au Québec, 35(3), 7-17.

Charbonneau, J. (2004). Contexte social et réversibilité des trajectoires au début de lâge adulte (document de recherche/ working paper): INRS (Urbanisation, Culture et Société).

Coren, E, Barlow, J., \& Stewart-Brown, S. (2003). The effectiveness of individual and group-based parenting programmes in improving outcomes for teenage mothers and their children: A systematic review. Journal of Adolescence, 26, 79-103.

Fox, K., Becker-Green, J., Gault, J., \& Simmons, D. (2005). Native American youth in transition: The path from adolescence to adulthood in two Native American communities. Portland, OR: National Indian Child Welfare Association.

Gauthier, M. (2000). Lâge des jeunes : Un fait social instable. Lien social et politiques - RIAC, 43, 23-32.

Girard, C., \& Lutumba Ntetu, A. (2004). La marge comme espace culturel. Territoire, identité et communauté chez les jeunes migrants innus (montagnais) et attikamekw au Québec. In G. Pronovost \& C. Royer (Eds.), Les valeurs des jeunes. Ste-Foy: Presses de l’Université du Québec.

Goyette, M., Turcotte, D., Mann-Feder, V., Grenier, S., Turcotte, M-Ė., Plagès, M., Pontbriand, A., Corneau, M. (2012). Évaluation de l'implantation et des effets d'interventions de groupe visant à soutenir le passage à la vie adulte de jeunes issus des centres jeunesse et de jeunes autochtones. Montréal, Les Cahiers de la CRÉVAJ, Cahier \#2012-03 CRÉVAJ05-12. 


\section{L'intervention de groupe pour soutenir le passage à la vie adulte des jeunes autochtone}

Goyette, M., Grenier, S., Pontbriand, A., Turcotte, M-E., El-Hadji, B., Mann-Feder, V. \& Turcotte, D., (2010). Rapport des résultats de la collecte de données auprès des jeunes (tome 2). Parcours d'entrée dans la vie adulte et stratégies d'autonomisation : une lecture dynamique des trajectoires de jeunes autochtones suivis ou placés en protection de la jeunesse. Montréal : Centre national de prévention du crime.

Goyette, M. et Grenier, S. (2008), Enjeux relatifs au passage à la vie adulte des jeunes autochtones : vers une meilleure compréhension de la particularité du contexte de communautés algonquines de l'Abitibi-Témiscamingue, Université du Québec en Abitibi-Témiscamingue et École National d’Administration publique.

Goyette, M., Bellot, C., \& Panet-Raymond, J. (2006). Le projet Solidarité Jeunesse. Dynamiques partenariales et insertion des jeunes en difficulté. Québec: Les Presses de l’Université du Québec.

Goyette, M., \& Turcotte, D. (2004). La transition vers la vie adulte des jeunes qui ont vécu un placement: un défi pour les organismes de protection de la jeunesse. Service social, 51(1), 30-44.

Graham, B. L. (2001). Resilience among American Indian youth: First Nations' youth resilience study. Graham, Barbara Leigh: University of Minnesota.

Grenier, S., Pontbriand, A., Lemay, A.-A., \& Goyette, M. (2010). D’Hier à demain. Programme d'intervention de groupe visant à faciliter le passage à la vie autonome des jeunes autochtones. UQAT : Rouyn-Noranda.

Guay, C. et Grammond, S. (2010). "À l'Écoute des peuples autochtones? : Le processus d'adoption de la "loi 125'”. Nouvelles pratiques sociales, 23 (1), 99-113.

Harris, M.B., \& Franklin, C.G. (2003). Effects of a cognitive-behavioral, school-based, group intervention with Mexican American pregnant and parenting adolescents. Social Work Research, 27 (2), 71-83.

Kissman, K. (1990). Parent skills training: Expanding school-based services for adolescent mothers. Research on Social Work Practice, 2 (2), 161-171.

La Prairie, C., \& Stenning, P. (2003). Exilés, rue principale : réflexions sur la sur-représentation autochtone dans le système de justice pénale. In Des gens d'ici: Les autochtones en milieu urbain (pp. 195-210). Ottawa: Projet de recherche sur les politiques. Gouvernement du Canada.

Loiselle, M. (2009). «La roue de bien-être : une contribution autochtone au travail social.» Intervention 131: 183-193.

Long, C., Downs, A., Gillette, B., In Sight, L., \& Konen, E. (2006). Assessing Cultural Life Skills of American Indian Youth. Child \& Youth Care Forum, 35(4), 289-304.

Mann-Feder, V., Roman, A., \& Corneau, M. (2009a). Soutien à la vie autonome. Le programme Droit devant. ENAP : Montréal.

Mann-Feder, V., Roman, A., \& Corneau, M. (2009b). Soutien à la vie autonome. Le programme Moi et cie.

Mann-Feder, V., Roman, A., \& Corneau, M. (2009c). Soutien à la vie autonome. Formation de base à la facilitation de groupe. Manuel. ENAP : Montréal.

McDonell, J.R., Limber, S.P., \& Connor-Godbey, J. (2007). Pathways teen mother support project: Longitudinal findings. Children and Youth Services Review, 29, 840-855.

Moyse Steinberg, D. (2008). Le travail de groupe. Un modèle axé sur l'aide mutuelle. Ste-Foy: Les Presses de l'Université Laval.

Munsell, G. T. (2004). Tribal Approaches to Transition. Tulsa, OK: The University of Oklaoma.

Schwarz, R., Davidson, A., Carlson, P., \& McKinney, S. (2005). The skilled facilitator fieldbook: Tips, tools, and tested methods for consultants, facilitators, managers, trainers, and coaches. San Francisco, CA: Jossey-Bass.

Stewart, S. (2009). Family Counseling as Decolinization : Exploring an Indigenous Social-Constructivist Approach in Clinical Practice. First Peoples Child \& Family Review, 4 (1), $62-70$.

Tweddle, A. (2007). Youth leaving care: How do they fare? New Directions for Youth Development, 113, 15-31. 